\title{
Peer-mentoring na educação em saúde: quais as suas aplicações, limitações e estratégias para o sucesso?
}

Peer-mentoring in the health education: what are its applications, limitations and strategies for success?

\author{
Mentoría de pares en educación para la salud: ¿cuáles son sus aplicaciones, limitaciones y \\ estrategias para el éxito?
}

Recebido: 11/08/2021 | Revisado: 16/08/2021 | Aceito: 19/08/2021 | Publicado: 22/08/2021

\author{
Natanias Macson da Silva ${ }^{1}$ \\ ORCID: https://orcid.org/0000-0003-1044-7319 \\ Universidade do Estado do Rio Grande do Norte, Brasil \\ E-mail: nataniassilva@alu.uern.br \\ Leonel Francisco de Oliveira Freire ${ }^{1}$ \\ ORCID: https://orcid.org/0000-0002-8734-5292 \\ Universidade do Estado do Rio Grande do Norte, Brasil \\ E-mail: leonelloliveira93@gmail.com \\ Anne Itamara Benigna Evangelista Aires ${ }^{1}$ \\ ORCID: https://orcid.org/0000-0002-9715-1088 \\ Universidade do Estado do Rio Grande do Norte, Brasil \\ E-mail: anneitamara@ hotmail.com \\ Marcos Daniel Oliveira e Silva ${ }^{1}$ \\ ORCID: https://orcid.org/0000-0003-2254-9398 \\ Universidade do Estado do Rio Grande do Norte, Brasil \\ E-mail: danielunp@gmail.com \\ Stheshy Vieira e Souza ${ }^{1}$ \\ ORCID: https://orcid.org/0000-0001-6868-7249 \\ Universidade do Estado do Rio Grande do Norte, Brasil \\ E-mail: stheshyvieira@alu.uern.br \\ Micássio Fernandes de Andrade ${ }^{2}$ \\ ORCID: https://orcid.org/0000-0003-2836-9080 \\ Universidade do Estado do Rio Grande do Norte, Brasil \\ E-mail: micassioandrade@uern.br \\ Ellany Gurgel Cosme do Nascimento ${ }^{3}$ \\ ORCID: https://orcid.org/0000-0003-4014-6242 \\ Universidade do Estado do Rio Grande do Norte, Brasil \\ E-mail: ellanygurgel@uern.br \\ Thales Allyrio Araújo de Medeiros Fernandes 4 \\ ORCID: https://orcid.org/0000-0003-4559-8918 \\ Universidade do Estado do Rio Grande do Norte, Brasil \\ E-mail: thalesallyrio@uern.br
}

\begin{abstract}
Resumo
Este estudo buscou investigar a contribuição do Peer-Mentoring na formação acadêmica de base e educação continuada de profissionais da saúde, bem como identificar as principais limitações, desafios e alternativas para atingir o sucesso de sua implementação. Trata-se de um estudo de revisão integrativa com busca sistemática, realizada nas bases de dados PubMed, Scopus, Web Of Science, Education Resources Information Center (ERIC) e Biblioteca Virtual em Saúde (BVS). Foram incluídos artigos eletrônicos, disponíveis na íntegra, nos idiomas português, inglês e/ou espanhol e publicados nos últimos 5 anos. Excluiu-se os estudos do tipo revisão de literatura, resumos, carta ao editor, manuscritos duplicados e estudos que não apresentaram relação com a temática. Os seguintes descritores foram combinados com os operadores booleanos AND e OR: "Peer-Mentoring”, "Peer Group", “Mentoring”, "Mentors", "Health Education" e "Education, Medical”. Foram coletados 31 artigos elegíveis, que passaram por uma análise na
\end{abstract}

\footnotetext{
${ }^{1}$ Mestrando(a) do Programa de Pós-Graduação em Saúde e Sociedade da Universidade do Estado do Rio Grande do Norte.

${ }^{2}$ Docente do Programa de Pós-Graduação em Saúde e Sociedade da Universidade do Estado do Rio Grande do Norte.

${ }^{3}$ Professora Adjunta IV da Universidade do Estado do Rio Grande do Norte e Coordenadora do Programa de Pós-Graduação em Saúde e Sociedade da Universidade do Estado do Rio Grande do Norte.

${ }^{4}$ Professor Adjunto IV da Universidade do Estado do Rio Grande do Norte e Docente do Programa de Pós-Graduação em Saúde e Sociedade da Universidade do Estado do Rio Grande do Norte
} 
íntegra e fichamento dos dados. Os estudos foram agrupados em cinco categorias de análise: (1) contribuição do PeerMentoring no processo de formação acadêmica; (2) impacto do Peer-Mentoring na educação continuada de profissionais da saúde educação continuada; (3) limitações são encontradas durante a execução do Peer-Mentoring; (4) desafios pedagógicos que devem ser vencidos, no transcurso da mentoria entre pares; (5) Como atingir o sucesso durante a implantação e execução de programas de Peer-Mentoring?. O Peer-Mentoring mostrou-se capaz de melhorar significativamente o processo de ensino em saúde. Perspectivas futuras apontam para uma maior adesão, por parte das instituições de ensino, em inserir a mentoria entre pares nos programas pedagógicos.

Palavras-chave: Educação em Saúde; Tutoria; Grupos de Pares; Capacitação; Aconselhamento.

\begin{abstract}
This study aimed to investigate the contribution of Peer-Mentoring in basic academic training and continuing education of health professionals, as well as to identify the main limitations, challenges and alternatives to achieve the success of its implementation. This is an integrative review study with systematic search, performed in PubMed, Scopus, Web Of Science, Education Resources Information Center (ERIC) and Virtual Health Library (VHL) databases. Electronic articles, available in full, in Portuguese, English and/or Spanish and published in the last 5 years, were included. Studies of the literature review type, abstracts, letter to the editor, duplicate manuscripts and studies that were not related to the theme were excluded. The following descriptors were combined with the Boolean operators AND and OR: "Peer-Mentoring", "Peer Group", "Mentoring", "Mentors", "Health Education" and "Education, Medical". Thirty-one eligible articles were collected, which underwent a full-text analysis and data fiching. The studies were grouped into five categories of analysis: (1) contribution of Peer-Mentoring in the academic training process; (2) impact of Peer-Mentoring on the continuing education of health professionals continuing education; (3) limitations are encountered during the execution of Peer-Mentoring; (4) pedagogical challenges that must be overcome, in the course of Peer-Mentoring; (5) How to achieve success during the implementation and execution of Peer-Mentoring programs? Peer-Mentoring proved to be able to significantly improve the health teaching process. Future perspectives point to a greater adherence by educational institutions to insert peer-mentoring in the pedagogical programs.
\end{abstract}

Keywords: Health Education; Preceptorship; Peer Group; Training; Counseling.

\title{
Resumen
}

Este estudio tuvo como objetivo investigar la contribución del Peer-Mentoring en la formación académica básica y la formación continua de los profesionales de la salud, así como identificar las principales limitaciones, desafíos y alternativas para lograr el éxito de su implementación. Se trata de un estudio de revisión integrador con una búsqueda sistemática, realizada en las bases de datos PubMed, Scopus, Web Of Science, Education Resources Information Center (ERIC) y Virtual Health Library (BVS). Se incluyeron artículos electrónicos, disponibles en su totalidad, en portugués, inglés y/o español y publicados en los últimos 5 años. Se excluyeron los estudios de tipo revisión de la literatura, los resúmenes, las cartas al editor, los manuscritos duplicados y los estudios que no estaban relacionados con el tema. Los siguientes descriptores se combinaron con los operadores booleanos AND y OR: "Peer-Mentoring", "Peer Group", "Mentoring", "Mentors", "Health Education" y "Education, Medical". Se recopilaron 31 artículos elegibles, que se sometieron a un análisis de texto completo y a una clasificación de datos. Los estudios se agruparon en cinco categorías de análisis: (1) contribución de la Tutoría entre pares en el proceso de formación académica; (2) impacto de la Tutoría entre pares en la formación continua de los profesionales de la salud; (3) limitaciones que se encuentran durante la ejecución de la Tutoría entre pares; (4) desafíos pedagógicos que deben ser superados, en el curso de la Tutoría entre pares; (5) ¿Cómo lograr el éxito durante la implementación y ejecución de los programas de Tutoría entre pares? La tutoría entre pares demostró ser capaz de mejorar significativamente el proceso de enseñanza de la salud. Las perspectivas de futuro apuntan a una mayor adhesión por parte de las instituciones educativas a la hora de insertar el Peer-Mentoring en sus programas pedagógicos.

Palabras clave: Educación en salud; Preceptoria; Grupos Paritario; Capacitación; Asesoramiento.

\section{Introdução}

Ao iniciar a jornada acadêmica no ensino superior, é cobrado do estudante uma capacidade adaptativa frente às mudanças intrínsecas ao processo de inserção no âmbito universitário. Dessa maneira, a depender da construção educacional de cada indivíduo, é inevitável que surjam problemas com os quais os discentes apresentem dificuldade em enfrentá-los (Franzoi \& Martins, 2020).

Nesse período de adaptação, a troca de experiências, conversas, apoio e suporte são atitudes que podem ser cruciais no enfrentamento de tais situações, principalmente no que se refere às questões acerca da saúde mental (Lemos et al., 2020). Inclusive, salienta-se que as graduações da área da saúde apresentam vários fatores estressores inerentes ao seu processo 
formativo, identificados, por vezes, nos seguintes "gatilhos": baixo rendimento acadêmico, esquecimento, insônia, irritabilidade, fadiga e outras queixas somáticas (Menezes et al., 2021).

Em contrapartida, sabe-se que a manutenção da saúde mental dos discentes é um dos pilares que garantem o sucesso no processo ensino-aprendizagem e, com isso, diversas alternativas pedagógicas têm sido apontadas para a temática em questão, sendo que estas podem variar de acordo com o método, objetivo e público alvo (Mota et al., 2019). Entre essas, destaca-se o mentoring, que é definido como uma metodologia de ensino que permite, aos alunos, a troca de experiências, compartilhamento de relatos e construção de um ambiente de ensino seguro (Lemos et al., 2020).

Com a sistematização do conhecimento, os programas de mentoring passaram a ser incorporados nas universidades dos Estados Unidos a partir de 1990, sobretudo nos cursos da área da saúde, com uma finalidade de melhorar a transmissão de conhecimento acadêmico (Franzoi \& Martins, 2020). Com o passar do tempo, percebe-se que também há uma transmissão de conhecimento pessoal, acadêmico e profissional - o que não está totalmente claro e foi alvo de investigação deste estudo de revisão.

É válido ressaltar que, apesar de ser uma metodologia contemporânea, o mentoring não é recente, já que no decorrer da história das civilizações, o aprendizado, tanto de sobrevivência quanto de ensino acadêmico, tem sido repassado dos sujeitos mais experientes para os menos experientes, acentuando a prerrogativa da passagem do conhecimento de maneira verticalizada (Menezes et al., 2021; Mota et al., 2019).

Entende-se que há vários modelos de mentoring, com organizações variadas e diferentes nomenclaturas, mas que mantêm a essência simples de "aprendizagem por pares", isto é, um aprendizado viabilizado por colegas de uma mesma categoria, possibilitando a troca do conhecimento de maneira horizontal e bidirecional (Olaussen et al., 2016).

Nesse cenário, o Peer-Mentoring (mentoria de pares, em uma tradução livre do inglês) surge como um desses modelos, caracterizado pela relação entre dois discentes de um mesmo curso ou categoria profissional (Akinla et al., 2018). O primeiro é o mentor, que geralmente situa-se em um período acadêmico mais avançado, enquanto que o segundo é o mentee (aluno iniciante ou mentorado) (Arthur \& Kram, 1985). Desse modo, o mentee é acompanhado pelo mentor através de encontros planejados de acordo com as necessidades e objetivos de ambas as partes (Akinla et al., 2018; Franzoi \& Martins, 2020).

Estudos mostram que essa metodologia de ensino pode oferecer o desenvolvimento de habilidades, aumento da produtividade e avanço na carreira acadêmica e/ou profissional (Farkas et al., 2019; Sambunjak et al., 2006; Weavind \& Jobin McGrane, 2018). Curiosamente, também tem sido relatado uma relação inversa entre o apoio emocional e social ofertado pela tutoria entre pares em detrimento de um menor sofrimento mental pelos acadêmicos (Pethrick et al., 2020). O que ainda não está claro é que condições são necessárias para obter os benefícios supracitados e se estas fazem parte de um consenso à luz da literatura. A exemplo, a orientação dos alunos tutores tem sido apontada, pela maioria do corpo docente de cursos de medicina dos Estados Unidos, como um dos fatores fundamentais para a execução da mentoria entre pares; o que faz um paradoxo com a realidade encontrada nesses sistemas: a orientação é rara ou inadequada (Farkas et al., 2019; Sambunjak et al., 2006; Weavind \& Jobin McGrane, 2018).

Em razão desses benefícios, instituições têm buscado implementar programas de mentoring que visem acolher e dar apoio a novos integrantes, gerando um processo significativo de aprendizado mútuo (Franzoi \& Martins, 2020). Todavia, a implantação de programas dessa natureza depende de fatores circunstanciais favoráveis, tanto da instituição e corpo docente quanto dos discentes envolvidos (Abrahamson et al., 2019), que serão investigados em diferentes cenários por esta revisão.

Esse estudo teve como objetivo investigar as atuais evidências sobre a contribuição do Peer-Mentoring na formação acadêmica de base e educação continuada de profissionais da saúde, bem como identificar as principais limitações, desafios e 
alternativas para atingir o sucesso de sua implementação em instituições de ensino e/ou serviços de saúde que possuem sistemas de educação continuada.

\section{Metodologia}

A revisão de literatura integrativa busca abordar lacunas de temas específicos a partir da síntese de múltiplos estudos, permitindo uma discussão abrangente sobre os métodos, resultados e conclusões dos manuscritos coletados (Priest, 2006; Soares et al., 2014). Nesse cenário, este estudo de revisão integrativa baseou-se na pergunta norteadora a seguir: "Há evidências científicas sobre a contribuição do Peer-Mentoring no processo ensino-aprendizagem na área da saúde (cursos de graduação, especializações e educação continuada em serviços de saúde)?”.

Com isso, realizou-se uma busca avançada e sistemática nas bases de dados: Medline (PubMed), Scopus (Elsevier), Web Of Science, Education Resources Information Center (ERIC) e Biblioteca Virtual em Saúde (BVS). Os critérios de inclusão para coleta dos manuscritos foram: (1) artigos eletrônicos, (2) disponíveis na íntegra, (3) nos idiomas português, inglês e/ou espanhol e, por fim, (4) publicados nos últimos 5 anos (2017 a 2021). Foram excluídos artigos do tipo revisão de literatura, resumos, carta ao editor, manuscritos duplicados e estudos que não apresentaram relação com a temática. Ademais, a busca sistemática foi realizada nos meses de maio e junho de 2021 e não considerou a literatura cinzenta. O processo de seleção, elegibilidade e inclusão dos artigos foi detalhado de acordo com a Declaração Prisma 2020 (Page et al., 2021).

Com base nos vocabulários controlados para indexação de artigos nas plataformas Mesh e Descritores em Ciências da Saúde (DeCS), foram definidos os descritores: "Peer-Mentoring”, "Peer Group", "Mentoring”, "Mentors", "Health Education" e "Education, Medical". Posteriormente, utilizou-se a ferramenta de pesquisa avançada das bases de dados supracitadas para a realização da estratégia de busca sistemática (vide material suplementar). Tais descritores foram combinados, entre si, utilizando os operadores booleanos AND e OR para fazer a interseção entre as linhas da estratégia de busca, resultando nas seguintes combinações: (1) Health Education AND Peer Group AND Mentoring OR Mentors; (2) Education, Medical AND Peer Group AND Mentoring OR Mentors; (3) Health Education OR Education, Medical AND PeerMentoring. Essas combinações foram rastreadas no título, resumo e assunto dos manuscritos, resultando em um total de 2.726 artigos.

De acordo com a aplicação dos critérios de inclusão e exclusão, obteve-se a coleta de 492 artigos, com posterior remoção dos estudos duplicados ( $n=156)$. Os manuscritos residuais $(n=336)$ foram submetidos ao processo de elegibilidade e extração de dados, por meio de uma revisão por pares, de modo independente e às cegas. Em seguida, deu-se a fase de exposição dos estudos incluídos por cada avaliador e definição da elegibilidade de cada artigo. Um terceiro avaliador foi requisitado para solucionar os casos de discordâncias. Por fim, a quantidade final de artigos coletados (n=57) esteve condicionada a um consenso entre os três revisores.

A partir disso, iniciou-se uma nova etapa de revisão por pares, às cegas, para a leitura dos resumos e/ou textos completos, o que resultou na eliminação de mais 26 artigos por incompatibilidade com os critérios de inclusão/exclusão. De acordo com o consenso dos três revisores, 31 artigos foram considerados elegíveis e alinhados com a pergunta norteadora deste estudo (Figura 1). Por fim, os manuscritos foram submetidos à leitura detalhada e à síntese de conteúdos por meio do instrumento de coleta de dados validado por Ursi (2006), com adaptação ao delineamento e objetivo deste estudo. 
Figura 1. Fluxograma da seleção e elegibilidade dos artigos.

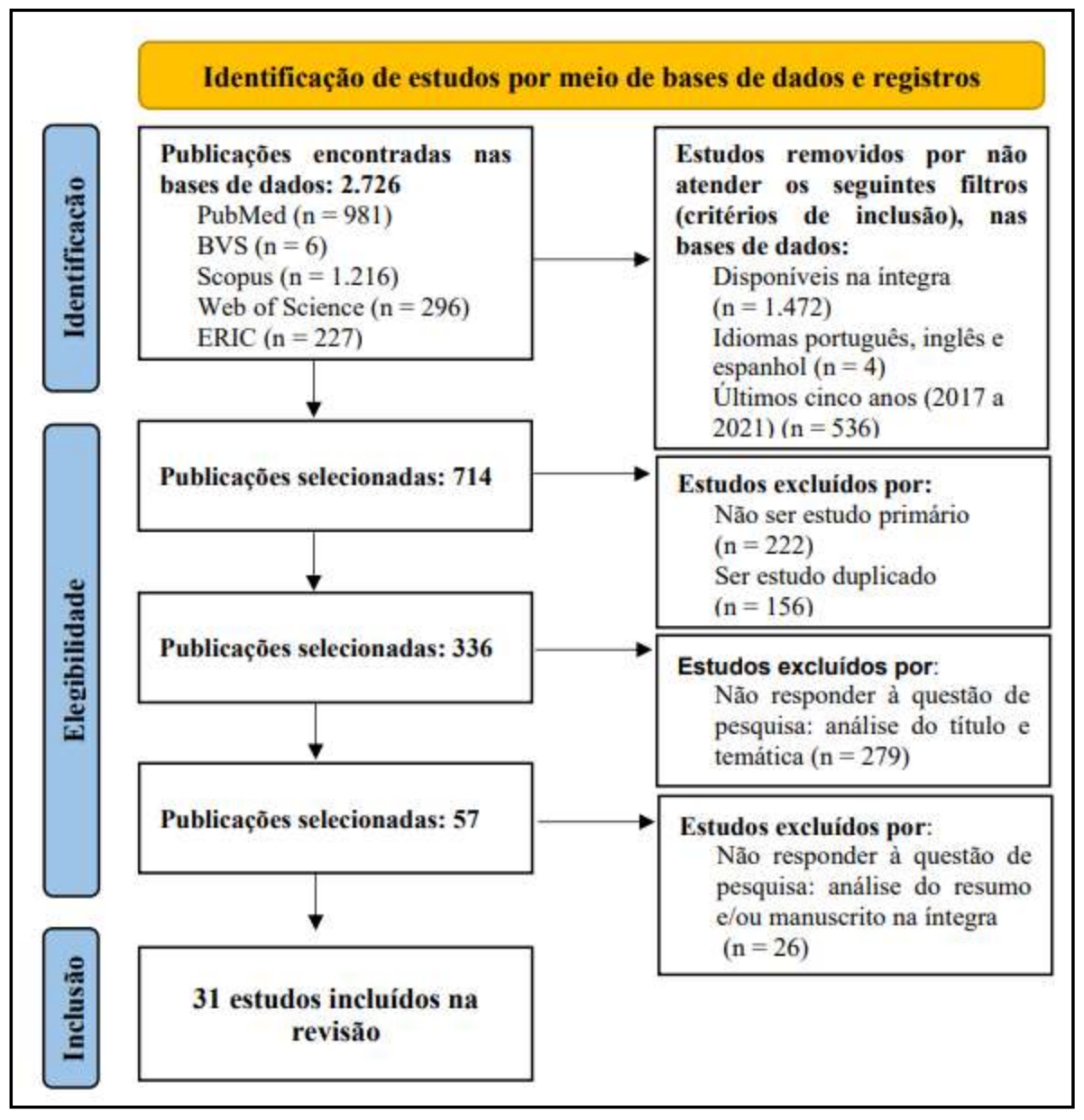

Legenda: BVS: Biblioteca Virtual em Saúde; ERIC: Education Resources Information Center. Fonte: Elaborado pelos Autores (2021).

\section{Resultados}

Após busca sistemática, 31 manuscritos foram submetidos a três análises sistemáticas (Tabela 1, 2 e 3, respectivamente) e alocados em cinco categorias de análise. A maioria dos estudos seguiu um delineamento metodológico com análise qualitativa $(n=23)$. O desenvolvimento pessoal, a segurança emocional e o crescimento nas relações interpessoais dos sujeitos constituíram as principais marcas do Peer-Mentoring.

Na Tabela 1, a primeira categoria buscou avaliar a contribuição do Peer-Mentoring no seio acadêmico, sobretudo em cursos de graduação e especialização ( $n=24$ estudos), enquanto que a segunda buscou avaliar o impacto dessa metodologia de ensino entre pares nos serviços de saúde que possuem programas de educação continuada ( $\mathrm{n}=7$ estudos).

A segunda análise (Tabela 2), estratificou 26 artigos em cada categoria: (1) limitações encontradas durante a execução do Peer-Mentoring e (2) desafios pedagógicos que devem ser vencidos para obtenção de um programa de ensino de qualidade. As limitações inerentes à aplicação do Peer-Mentoring, bem como os desafios para obter sucesso com sua inserção, no cenário de ensino em saúde, foram descritas por parte substancial dos autores.

Por fim, a última análise sistemática (Tabela 3) se deteve a categoria "Como atingir o sucesso durante a implantação e execução de programas de Peer-Mentoring?", que permitiu desenvolver uma síntese sobre as principais estratégias para atingir o sucesso em programas de ensino $(n=26$ estudos $)$. 
Tabela 1. Caracterização sistemática da contribuição do Peer-Mentoring no processo ensino-aprendizagem na educação de base e continuada em serviços de saúde.

EIXO TEMÁTICO

Contribuição do PeerMentoring no processo de formação acadêmica

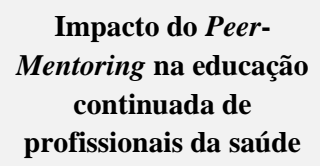

\section{CARACTERIZAÇÃO}

Os estudos, agrupados neste eixo temático, avaliam a importância da inserção do PeerMentoring no processo de formação acadêmica, em diferentes cursos da área da saúde, bem como o impacto na formação dos estudantes, tanto para fins acadêmicos e profissionais quanto para desenvolvimento pessoal.

Chambers et al. (2020 Crisp et al. (2020); Cross et al. (2020); Franzoi \& Martins (2020); Krishna et al. (2020); Loda et al. (2020.a); Loda et al. (2020.b); Ntho, Pienaar \& Sehularo (2020); Rompolski \& Dallaire (2020); Souza, Reato \& Bellodi (2020); Patel et al. (2021); Zibold et al. (2021)

\section{CONCLUSÃO}

Trata-se de um eixo temático profusamente discutido por vários autores, evidenciando o importante papel do PeerMentoring no processo acadêmico, possibilitando:

1- Complementação do conteúdo tradicional ofertado nos cursos de saúde;

2- Aquisição otimizada do conhecimento teórico e prático;

3- Ganhos de habilidades procedimentais (ex.: técnica cirúrgica);

4- Rápida abordagem de soluções problemas e/ou dificuldades de ensino teórico-prático;

5- Suprir a falta de tutores sêniores, com a mentoria entre pares (alguns estudos mostram superioridade do PeerMentoring, como estratégia de ensino prático);

6- Aos tutores, o desenvolvimento de habilidades pedagógicas, de liderança e reconhecimento situacional em relação às falhas mais frequentes, encontradas durante o processo;

7- Construção de vínculos interpessoais e de um ambiente igualitário e seguro, resultando em um aprendizado horizontalizado, aos pares, que preza pela autoconfiança (e não pelo julgamento);

8- Liberdade de escolha, ao aderir programas de PeerMentoring de modo eletivo, bem como benefícios emocionais e sociais;

9- Trocas de experiências em contextos socioculturais diferentes, diversificando as relações mentor-mentee;

10- Melhora do currículo profissional;

11- Ótima relação médico-paciente, em projeções futuras, com uma práxis de natureza reflexiva e relacional.

Phipps et al. (2018); Smith et al. (2018); Masterson Creber et al. (2019); Prunuske, Houss \& Kosobuski (2019); Blake et al. (2020); Franzoi \& Martins (2020); Hossain et al. (2021)
Esse eixo temático reuni estudos que verificaram o processo de inserção do Peer-Mentoring e a seu impacto

desenvolvimento de competências e habilidades de profissionais de saúde em diferentes cenários.

A utilização da aprendizagem entre pares iniciou a partir da oferta reduzida de profissionais experientes para a capacitação da equipe. Após a aplicação dessa metodologia de ensino, os profissionais passaram a trocar experiências.
Os profissionais atuantes passaram a treinar funcionários recém chegados em seus locais de atuação. Em suma, o Peer-Mentoring permitiu:

1- Complementação do conteúdo tradicional ofertado nos cursos de pós-doutorado e residências médicas;

2- Suporte acadêmico (melhora dos conhecimentos teóricos e procedimentais), emocional e pessoal, tanto ao mentor quanto ao aluno;

3- Educação continuada de profissionais em serviços de assistência à saúde;

4- Aquisição de habilidades necessárias ao profissional formado, como o perfil de liderança, e competências centrais para a prática colaborativa interprofissional, com a melhora da execução das funções e responsabilidades, comunicação, coordenação, cooperação e trabalho em equipe;

5- Aumentar potencialmente as competências de profissionais em cenários como: imunização contra o SarsCov-2 e manejo especializado de pacientes oncológicos.

Fonte: Elaborado pelos Autores (2021). 
Tabela 2. Análise sistemática das limitações do Peer-Mentoring e dos desafios pedagógicos durante a sua execução em instituições de ensino e serviços de saúde.

\section{EIXO TEMÁTICO}

Quitações são encontradas durante a execução do PeerMentoring?

Quais os desafios pedagógicos que devem ser vencidos, no transcurso da mentoria entre pares?

\section{ESTUDOS}

Lewis et al. (2016); Engels et al. (2018); Lapp, Makowka \& Recker (2018); Phipps et al. (2018); Smith et al. (2018); Tamachi et al. (2018); Wareing et al. (2018); Abrahamson et al. (2019); Celebi et al. (2019); González et al. (2019); Hsiang-Te Tsuei et al. (2019); Masterson Creber et al. (2019); Prunuske, Houss \& Kosobuski (2019); Scott et al. (2019); Blake et al. (2020); Crisp et al. (2020); Cross et al. (2020); Krishna et al. (2020); Loda et al. (2020.a); Loda et al. (2020.b); Ntho, Pienaar \& Sehularo (2020); Rompolski \& Dallaire (2020); Souza, Reato \& Bellodi (2020); Hossain et al. (2021); Patel et al. (2021); Zibold et al. (2021)

Lewis et al. (2016); Engels et al. (2018); Lapp, Makowka \& Recker (2018); Phipps et al. (2018); Smith et al. (2018); Tamachi et al. (2018); Wareing et al. (2018); Abrahamson et al. (2019); Celebi et al. (2019); González et al. (2019); Hsiang-Te Tsuei et al. (2019); Masterson Creber et al. (2019); Prunuske, Houss \& Kosobuski (2019); Scott et al. (2019); Blake et al. (2020); Crisp et al. (2020); Cross et al. (2020); Krishna et al. (2020); Loda et al. (2020.a); Loda et al (2020.b); Ntho, Pienaar \& Sehularo (2020); Rompolski \& Dallaire (2020); Souza, Reato \& Bellodi (2020); Hossain et al. (2021); Patel et al. (2021); Zibold et al. (2021)

\section{CARACTERIZAÇÃo}

Diante de um copilado de dados desses estudos, esse eixo temático mostra as principais limitações inerentes à implementação da metodologia "PeerMentoring" no processo de ensino em saúde, seja na graduação, pós-graduação ou no aperfeiçoamento de funcionários atuantes em serviços de saúde.

Esses estudos investigaram as principais estratégias organizacionais que resultaram na eficiência do programa de Peer-Mentoring. Nesse processo, os autores identificaram os desafios pedagógicos que devem ser vencidos durante a mentoria entre pares.
Em relação aos desafios, os estudos apontam para a falta de planejamento das atividades, de empenho e de apoio proveniente dos docentes. Além disso, as falhas na comunicação e acessibilidade aos mentores, bem como a posição de superioridade apresentadas por estes foram citadas como pontos negativos que podem comprometer o sucesso dos resultados. Outros desafios foram elencados:

a) Construção de uma periodicidade e dedicação com horários reservados para a mentoria;

b) Ensino sem imposições de objetivos pessoais do mentor ao mentee;

c) Diferenças socioculturais, de idade e/ou maturidade, pois podem gerar conflitos;

d) Baixo sentimento de pertencimento ao ambiente acadêmico e/ou de ensino;

e) Falta de feed-back sobre as contribuições do mentor ao mentorado e vice-versa;

f) Risco de gerar impactos negativos nos participantes, como frustrações, fragilidades na confiança, na autoestima e no envolvimento;

g) Risco de confusões no processo de ensino inerentes à não sincronia entre as informações transmitidas pelos tutores sêniores e tutores entre pares;

h) Situações que prejudiquem o relacionamento, envolvimento e encontros práticos, como visto durante a Pandemia pela COVID-19. 
Tabela 3. Análise sistemática das estratégias necessárias para atingir o sucesso na implantação de programas de PeerMentoring em instituições de ensino e serviços de saúde.

\section{ESTUDOS}

Lewis et al. (2016); Engels et al. (2018); Lapp, Makowka \& Recker (2018); Phipps et al. (2018); Smith et al. (2018); Tamachi et al. (2018); Wareing et al. (2018); Abrahamson et al. (2019); Celebi et al. (2019); González et al. (2019); Hsiang-Te Tsuei et al. (2019); Masterson Creber et al. (2019); Prunuske, Houss \& Kosobuski (2019); Scott et al. (2019); Blake et al. (2020); Crisp et al. (2020); Cross et al. (2020); Krishna et al. (2020); Loda et al. (2020.a); Loda et al. (2020.b); Ntho, Pienaar \& Sehularo (2020); Rompolski \& Dallaire (2020); Souza, Reato \& Bellodi (2020); Hossain et al. (2021); Patel et al. (2021); Zibold et al. (2021)
Como atingir o sucesso durante a implantação e execução de programas de Peer-Mentoring?

Os autores alocados no terceiro eixo entendem que o Peer-Mentoring deve ser rigorosamente planejado, de acordo com os recursos da instituição e demandas dos alunos, seguindo as seguintes estratégias para o sucesso:

1) Capacitações iniciais e contínuas, de modo longitudinal, além do suporte docente aos mentores;

2) Acompanhamento do progresso dos participantes (mentor e mentee) durante as sessões de tutoria entre pares, por meio de avaliações apropriadas e específicas para cada objetivo de aprendizagem e/ou atuação. Essas avaliações devem ocorrer em momentos oportunos, de maneira longitudinal, holística e de fácil acessibilidade; e, desse modo, permitirão o diagnóstico de intercorrências e planejamento de adaptações futuras;

3) Compreender as expectativas dos mentores e mentees;

4) Garantir a construção de um relacionamento íntimo entre o mentor e o mentee, bem como uma boa organização dos encontros;

5) Considerar o nível de aprendizagem do mentee durante o planejamento dos conteúdos;

6) Em casos de prejuízo às práticas presenciais, como ocorreu em alguns programas de mentoria entre pares, por causa da pandemia pela COVID-19, recomenda-se: adaptações ao programa de mentoria, com um planejamento personalizado e adaptado ao sistema de ensino remoto, mediante uso de tecnologias da informação e comunicação. Além disso, as peculiaridades e necessidades dos mentores e mentees devem ser sempre consideradas durante a elaboração do plano adaptativo.

Fonte: Elaborado pelos Autores (2021).

\section{Discussão}

De acordo com os estudos coletados nesta revisão, o Peer-Mentoring viabiliza a formação de relações acadêmicas humanizadas ao estabelecer o vínculo entre discentes menos e mais experientes (Abrahamson et al., 2019; Chambers et al., 2020; Crisp et al., 2020; Cross et al., 2020; Franzoi \& Martins, 2020; González L et al., 2019; Rompolski \& Dallaire, 2020; Souza et al., 2020). Os discentes mais avançados, quando colocados a ensinar, são induzidos a aprender mais sobre si mesmos e sobre os conteúdos ministrados, contribuindo para a percepção do espírito de cooperação e obtenção de habilidades de liderança.

Por meio dos autores, os alunos que decidem serem mentores veem, nessa atividade, uma oportunidade de desenvolver habilidades acadêmicas sólidas, melhorar relação médico-paciente, além de aprimorar seus currículos profissionais (Abrahamson et al., 2019; Chambers et al., 2020; Crisp et al., 2020; Cross et al., 2020; Franzoi \& Martins, 2020; González L et al., 2019; Patel et al., 2021; Rompolski \& Dallaire, 2020; Souza et al., 2020).

Estudos corroboram com esse evento ao afirmarem que o desenvolvimento de habilidades de ensino, comunicação oral, bem como melhor apreensão de conhecimento sobre os conteúdos ensinados foi significativamente maior nos indivíduos que experimentaram a mentoria (Evans \& Cuffe, 2009; Naeger et al., 2013). Particularmente, a adesão à mentoria entre pares pode ser mais intensa nos primeiros períodos da graduação, o que pode ser explicado pelas várias adaptações ao ambiente universitário e maior tempo livre, quando comparado a períodos avançados e estágios práticos (Lapp et al., 2018).

Outra importante contribuição do método Peer-Mentoring diz respeito à fluidez do processo de aprendizagem (Loda, Erschens, Nikendei, Giel, et al., 2020; Rompolski \& Dallaire, 2020), facilitada pela liberdade em que o mentee possui em opinar sobre os objetivos (Krishna et al., 2020), suas peculiaridades e escolha dos conteúdos que mais sente dificuldade (Engels et al., 2018). Além disso, essa maleabilidade garanti maior proximidade estabelecida entre os pares e a criação de valiosos momentos de troca de experiências. Prova disso, o mentor, que passou pelas mesmas experiências com sucesso, pode 
antecipar as dificuldades de aprendizagem e compreender os prováveis desafios cognitivos dos mentorados (Lockspeiser et al., 2008; Ten Cate \& Durning, 2007).

O processo de ensino por meio do Peer-Mentoring também se baseia na empatia entre tutores e alunos, gerando o que os autores denominam de "congruência social" (Loda, Erschens, Nikendei, Giel, et al., 2020; Loda, Erschens, Nikendei, Zipfel, et al., 2020). Esta parece representar a base de um relacionamento sólido e transparente, que culmina em um cenário confortável e convidativo. Este ambiente impulsiona os mentees a explorarem as lacunas da aprendizagem, permitindo um diagnóstico rápido das fragilidades e planejamento de ações resolutivas por parte do mentor.

Nesse sentido, detectamos um consenso entres os autores: o ambiente de aprendizagem, estabelecido no PeerMentoring, visa a criação de espaços agradáveis de aprendizagem onde a transmissão do conhecimento ocorre de forma afetiva (Celebi et al., 2019; Green, 2018; Lockspeiser et al., 2008; Scott et al., 2019). Outro importante fator positivo, relatado por esses autores, foi a fácil acessibilidade aos tutores, gerando uma relação de segurança e auto confiança entre os mentor-mentee. Quando este acesso é dificultado pode haver um comprometimento significativo da metodologia de ensino (Ntho et al., 2020); e por isso, em situações de isolamento social, como vivenciado pela COVID-19, faz-se necessário traçar estratégias que garantam maior proximidade dos pares (Zibold et al., 2021). Curiosamente, as habilidades e atributos dos mentores relacionado ao apoio psicossocial pode ser o aspecto mais importante para maior desenvolvimento dos mentees, especialmente para discentes inexperientes (Terrion \& Leonard, 2007).

Nesse constructo, o espírito de competição e o exercício de julgamento dos colegas é praticamente abolido, propiciando espaços de compreensão, comunicação efetiva e companheirismo na troca de experiências.

À luz da literatura analisada, reconhecemos que essa metodologia de ensino vem ganhando espaço entre as práticas de ensino em diferentes instituições produtoras de conhecimento. Com base nos estudos elencados, a mentoria entre pares dispõe de inúmeras possibilidades de apreensão de habilidades e competências por parte de alunos e também de seus mentores. Porém, existem aspectos ainda pouco explorados no que diz respeito ao impacto produzido para a organização curricular institucional e para a prática docente tradicional. Faz-se necessária a realização de novos estudos apontando esses aspectos.

Os resultados coletados nos estudos se mostraram bastante significativos, no tocante à apropriação de conceitos acadêmicos (teóricos e/ou práticos) pelos mentores e mentees e às outras possiblidades que o Peer-Mentoring pode proporcionar a esses atores do processo ensino-aprendizagem, impactando-os positivamente em diferentes cenários.

Em primeira instância, é válido ressaltar que o papel desempenhado pelo mentor não se assemelha ao do professor ou preceptor atuante na educação continuada em serviços de saúde. Nesse seguimento, destaca-se o papel da metodologia induzir a criação de um espaço propício ao raciocínio crítico-reflexivo e o desenvolvimento de habilidades como autonomia, comunicação, gestão, confiança e independência, tanto em nível pessoal quanto profissional (Franzoi \& Martins, 2020).

Um estudo realizado por Masterson Creber e colaboradores (2019) verificou um aumento no desenvolvimento de habilidades como gestão, liderança e um grande avanço na carreira dos mentorados (Masterson Creber et al., 2019). Esta pesquisa foi realizada entre estagiários de pós-graduação e o corpo docente júnior, com o objetivo de avaliar um programa de mentoria por pares; com isso, viu-se que a metodologia facilitou a interação interprofissional, o estabelecimento de uma rede de colaboração de pesquisas e o aumento de publicações e concessão de bolsas (Masterson Creber et al., 2019).

Outros cenários foram apresentados pelos estudos elencados nesta revisão, como treinamento de profissionais atuantes em centros especializados no tratamento de cânceres (Phipps et al., 2018), especialidade médica em ultrassonografia (Smith et al., 2018), pós-graduação e pesquisa biomédica (Lewis, Martina, McDermott, Chaudron, et al., 2017), residência médica em Medicina de Família e Comunidade (Prunuske et al., 2019), serviços de saúde com atendimento de clínica médica e medicina do trabalho (Blake et al., 2020) e, por fim, em programas de assistência à saúde e imunização (Hossain et al., 2021). 
Diante desses cenários, os resultados mostram que o sucesso da mentoria por pares em um contexto profissional se dá pela troca de experiência e o desenvolvimento de habilidades específicas, sendo necessárias para o sucesso da profissão.

A aprendizagem continuada na prática trabalhista tem como objetivo a melhoria das funções do servidor; contudo, o mentor (profissional mais experiente) coloca-se na mesma posição do aluno (profissional júnior), promovendo uma troca de conhecimentos pautada na horizontalidade (Prunuske et al., 2019).

Um estudo, realizado por Smith e colaboradores, verificou que o método de ensino entre pares foi eficiente e permitiu um aprimoramento das habilidades de liderança, comunicação e organização (Smith et al., 2018). Esses resultados foram conseguidos após avaliação de um treinamento organizado pelos docentes, os quais orientaram médicos residentes de um curso de ultrassonografia diagnóstica para atuarem como treinadores/professores de discentes que estavam no primeiro ano de residência (Smith et al., 2018).

A limitação da quantidade de docentes e capacitações aos mentores parece ser ainda mais desafiadora nos serviços de saúde. Para suprir essas demandas, o sistema de mentoria entre pares tem sido uma ferramenta útil, adotada por algumas instituições.

Um estudo realizado por Phipps e colaboradores (2018) buscou avaliar um programa de mentoria entre pares em um serviço de saúde especializado em oncologia, iniciado pela necessidade de orientar cientistas juniores com seus projetos de pesquisas e, em contrapartida, pelo déficit no número de mentores sêniores (Phipps et al., 2018). Desse modo, foi criado um ambiente de aprendizagem, empoderamento e responsabilidade do grupo, que foi avaliado após dois anos de experiência. Viuse que os profissionais inseridos inicialmente na prática passaram a treinar os novos grupos recém chegados, alcançando, assim, todos os objetivos traçados no início do programa de Peer-Mentoring.

A exemplo desse último estudo, percebe-se que programas de Peer-Mentoring são funcionantes a longo prazo e podem contribuir sobremaneira com o desenvolvimento do corpo técnico-científico da instituição, de modo multiprofissional e integrado.

A aprendizagem interprofissional tem um papel inovador em ambientes de trabalho, uma vez que proporciona uma troca de diferentes experiências profissionais (Blake et al., 2020). Os mesmos autores observaram o crescimento acadêmico e profissional de estudantes da área da saúde, ao vivenciarem as nuances do "mundo real" em um serviço de promoção à saúde. Acompanhados por uma equipe de profissionais experientes, os estagiários obtiveram uma aprendizagem interdisciplinar, bem como o desenvolvimento das aptidões dos estagiários em cinco áreas estudadas, sendo elas: colaboração, coordenação, cooperação, comunicação e elogio. Por outro lado, houve significativa evolução de habilidades dos profissionais, que atuaram como mentores entre pares engajados no ensino dos estagiários iniciantes (Blake et al., 2020).

Esse crescimento mútuo também é reconhecido por outros autores. Para Franzoi \& Martins (2020), o programa de mentoring permite maior acolhimento e aprendizado de várias áreas de atuação, aumentando as possibilidades de inserção no mercado de trabalho acadêmicos do curso de enfermagem e recém formados. O programa, avaliado por estes autores, contribuiu para o desenvolvimento da integração interprofissional, engajamento, humanização e a importância do trabalho coletivo (Franzoi \& Martins, 2020).

Precisamos destacar, ainda, que a mentoria entre pares também mostrou-se eficiente quando utilizada no plano de ação global para vacinação contra o Sars-Cov-2 (Hossain et al., 2021). Nesse estudo, as estratégias de aprendizagem inovadoras foram realizadas por meio do aplicativo WhatsApp, permitindo a realização de treinamentos e, por conseguinte, o aumento da resolutividade na prática médica (Hossain et al., 2021). Ainda, os mentores forneceram apoio para os seus mentorados identificarem os problemas individuais e desafios; com isso ajudaram no avanço do desenvolvimento de habilidades, promovendo uma discussão e troca de conhecimentos sobre as experiências na prestação de serviços de 
imunização (Hossain et al., 2021). Tanto os mentores como os mentees participavam de um único grupo, possibilitando uma construção de relacionamento mais próximo (Hossain et al., 2021).

De acordo com os estudos alocados nesse eixo de discussão, podemos concluir que o Peer-Mentoring é uma ferramenta de ensino útil, haja vista o seu forte potencial em estimular o aprimoramento profissional, técnico-científico e avanços na carreira dos trabalhadores da área da saúde.

Ademais, o Peer-Mentoring possui características únicas, como a fluidez em adaptar-se a diferentes cenários, como o distanciamento social. Nessas situações, sugerimos o uso de tecnologias de informação e comunicação, como redes sociais, de modo a prosseguir com o programa garantindo a proximidade desejada entre o mentor e o mentee.

Ao analisar as narrativas dos diversos autores, é possível perceber limitações e desafios inerentes à implementação da tutoria entre pares, assim como avanços de sua prática; o que evoca a necessidade de uma reflexão crítica sobre tais circunstâncias, uma vez que estas norteiam a conduta de mentores e mentees e o sucesso da metodologia de ensino.

As principais dificuldades relatadas pelos mentores são relacionadas às expectativas, objetivos e exercícios de funções (Rompolski \& Dallaire, 2020; Souza et al., 2020). Nesse cenário, o surgimento de questionamentos no início do processo de mentoria é amplamente aceitável e esperado, porém, para os mentores, isso parece gerar preocupações, ansiedade ou até mesmo incertezas em relação às suas habilidades pessoais para administrar as reuniões. Em razão disso, sugerimos um exercício da mentoria fundamentado em comunicação, transparência e diálogo entre os mentores, mentees e docentes; e esse é apenas um dos passos para obter uma boa condução do Peer-Mentoring.

Itens como motivação, amplo conhecimento e experiência como professor, monitor ou pesquisador não são suficientes para ser um bom mentor ou estabelecer uma relação de mentoria satisfatória (Loda, Erschens, Nikendei, Giel, et al., 2020; Loda, Erschens, Nikendei, Zipfel, et al., 2020; Masterson Creber et al., 2019). Para além disso, faz-se necessário capacitações longitudinais (e não somente iniciais) (Abrahamson et al., 2019; Celebi et al., 2019; Lewis, Martina, McDermott, Trief, et al., 2017; Patel et al., 2021; Prunuske et al., 2019; Scott et al., 2019; Smith et al., 2018; Tamachi et al., 2018; Tsuei et al., 2019; Wareing et al., 2018), bem como a coleta de feedbacks para o melhoramento do programa de ensino (Hossain et al., 2021). Com essas estratégias, entendemos que o planejamento e execução dos encontros serão otimizados e dificilmente haverá desvios em relação aos objetivos da metodologia.

Ressaltamos que o treinamento contínuo dos mentores pode ser decisivo para experiências exitosas no desenvolvimento da mentoria entre pares. Inclusive, no transcurso das reuniões, o mentorado deve ser estimulado a trazer demandas que evocam a necessidade de uma abordagem diferenciada e íntima, de modo síncrono com as peculiaridades do mentee (Abrahamson et al., 2019; Loda, Erschens, Nikendei, Giel, et al., 2020; Loda, Erschens, Nikendei, Zipfel, et al., 2020; Scott et al., 2019).

O reconhecimento das particularidades do mentorado exige uma alta sensibilidade do mentor, que se constrói a partir de um relacionamento íntimo. Este, por sua vez, é um dos desafios relatados, uma vez que o distanciamento interpessoal pode implicar na redução da assiduidade nas reuniões (Crisp et al., 2020; Cross et al., 2020; Engels et al., 2018). Contudo, quando há clareza nos objetivos dos encontros, concordância e compreensão do mentor, mentee e instituição proponente, tem-se como resultado uma maior aquisição de habilidades interpessoais, acadêmicas e profissionais (Lewis, Martina, McDermott, Trief, et al., 2017; Tamachi et al., 2018; Tsuei et al., 2019; Wareing et al., 2018).

De modo singular, quanto mais o mentorado adquire confiança, mais independente ele se torna em relação ao mentor; retratando o seu crescimento intelectual e pessoal (Lapp et al., 2018; Zibold et al., 2021), além da possibilidade de o mentor deixar de ser "guia" e transformar-se em um colega ou mesmo amigo pessoal (Patel et al., 2021; Prunuske et al., 2019; Scott et al., 2019; Smith et al., 2018). 
Outro ponto de discussão destacado nos estudos é a falta de tempo para um manejo adequado das atividades acadêmicas, profissionais, cotidianas e aquelas relacionadas à mentoria entre pares (Loda, Erschens, Nikendei, Giel, et al., 2020; Loda, Erschens, Nikendei, Zipfel, et al., 2020; Masterson Creber et al., 2019). Obviamente, para o sucesso do programa de ensino de mentoria entre pares, faz-se necessário investir tempo, associado a um bom planejamento (Crisp et al., 2020; Cross et al., 2020; Engels et al., 2018; González L et al., 2019; Krishna et al., 2020); do contrário, o estabelecimento de vínculos interpessoais (uma das fortes características dessa metodologia) e o crescimento acadêmico e/ou profissional serão fragilizados.

Estudos mostram que alguns mentores investem mais tempo no desenvolvimento das relações interpessoais, enquanto outros focam nos encontros para fins acadêmicos e desenvolvimento de habilidades técnicas e, por fim, existem mentores que exploram predominantemente os fatores emocionais e intuitivos (Rompolski \& Dallaire, 2020; Souza et al., 2020). Esses comportamentos são modulados pelas personalidades de cada mentor. Nesta revisão, não detectamos estudos que abordam a influência de personalidade na tutoria entre pares, porém, a autoconsciência do mentor, a identificação do seu próprio estilo e o comprometimento em desenvolver e manter um relacionamento a longo prazo com seu mentorado parece ser fortes pilares no processo ensino-aprendizagem.

As divergências socioculturais e de idade são elencadas como fatores que podem ser decisivos na criação de vínculos entre as partes (Blake et al., 2020; Ntho et al., 2020; Phipps et al., 2018). Por isso, recomendamos que durante o processo de capacitações longitudinais essas questões sejam exploradas.

Para que ocorra êxito na aplicabilidade do Peer-Mentoring, etapas básicas devem ser seguidas de maneira pontual e contínua, afim de reforçar elos entre as instituições e os mentores, os mentores e os mentees e entre os mentees e as instituições de ensino (Abrahamson et al., 2019; Celebi et al., 2019). As principais estratégias sinalizadas nos estudos foram resumidas na Tabela 3.

Destacamos que a estratégia de capacitações na fase inicial já é perceptível em algumas instituições, porém, na grande maioria há ainda a necessidade de treinamento contínuo com os mentores e acompanhamento dos mentorados (Rompolski \& Dallaire, 2020; Souza et al., 2020), haja visto que os mesmos estão expostos a determinadas situações que requerem opiniões, posicionamentos e habilidades bem elaboradas. Uma ferramenta que possibilite feedback pode ser utilizada com a finalidade de reconhecer possíveis intercorrências e traçar planos de ações específicas.

Ademais, sabendo que o encontro presencial é uma forte característica do Peer-Mentoring, Zibold e colaboradores (2021) estudaram as estratégias e modificações necessárias para manter um programa de mentoria entre pares durante a pandemia pela COVID-19 (Zibold et al., 2021). Nesse caso, os autores viram que a proposta de encontros online induziu o desenvolvimento de forte elo entre mentores e mentees (Zibold et al., 2021). Assim como esses autores, recomendamos que o programa de mentoria entre pares seja fluido e capaz de adaptar-se a diversos cenários, incluindo situações onde o encontro presencial não seja possível.

Por fim, reconhecemos que o sucesso do programa depende de vários passos que precisam ser seguidos. Contudo, quando bem planejados, os programas apresentam forte potencial em promover sucesso acadêmico, profissional e pessoal.

\section{Conclusão}

A literatura explorada no presente estudo revela o Peer-Mentoring como uma importante ferramenta no processo de ensino-aprendizagem, tanto no meio acadêmico, quanto no âmbito profissional. Isso porque a construção de um ambiente empático, leve e fluido, propiciado pela mentoria entre pares, contribui para a troca de experiências e saberes. 
A relação horizontal, estabelecida entre o binômio mentor e mentorado, promove à redução da sensação de julgamento e competição, presente no ambiente de ensino tradicional; com isso, o discente externaliza as suas fragilidades com maior facilidade, o que o faz ascender na aquisição de conhecimentos.

De maneira conjunta, o mentor também é beneficiado com a ferramenta. Este, desenvolve várias habilidades, como liderança e docência, adquirindo uma postura prudente e profissional, ainda no ambiente acadêmico.

Para além da esfera acadêmica, a prática de mentoria permite a troca de saberes entre profissionais com diferentes níveis de experiência, por vezes dentro de uma mesma equipe, favorecendo o aprimoramento de técnicas e o ensino continuado em serviços de saúde.

As potencialidades da mentoria entre pares, abordadas nessa revisão, reverberam expectativas futuras que apontam para a maior adesão desta ferramenta por instituições de ensino e serviços de saúde com educação continuada.

Por fim, em trabalhos futuros, sugerimos o desenvolvimento e avaliação de novas estratégias pedagógicas que tenham como finalidade otimizar o processo de implantação de programas de Peer-Mentoring em instituições de ensino superior e em serviços de saúde com educação continuada.

\section{Referências}

Abrahamson, E. D., Puzzar, C., Ferro, M. S., \& Bailey, S. (2019). Peer Mentors' Experiences and Perceptions of Mentoring in Undergraduate Health and Sports Science Programmes. Journal of Pedagogical Research, 3(2), 21-37.

Akinla, O., Hagan, P., \& Atiomo, W. (2018). A systematic review of the literature describing the outcomes of near-peer mentoring programs for first year medical students. BMC Medical Education, 18(1), 98. https://doi.org/10.1186/s12909-018-1195-1

Arthur, M. B., \& Kram, K. E. (1985). Mentoring at Work: Developmental Relationships in Organizational Life. Administrative Science Quarterly, 30(3), 454. https://doi.org/10.2307/2392687

Blake, H., Somerset, S., Whittingham, K., Middleton, M., Yildirim, M., Evans, C., Blake, H., Somerset, S., Whittingham, K., Middleton, M., Yildirim, M., \& Evans, C. (2020). WHIRL Study: Workplace Health Interprofessional Learning in the Construction Industry. INTERNATIONAL JOURNAL OF ENVIRONMENTAL RESEARCH AND PUBLIC HEALTH, 17(18).

Celebi, N., Griewatz, J., Malek, N. P., Hoffmann, T., Walter, C., Muller, R., Riessen, R., Pauluschke-Fröhlich, J., Debove, I., Zipfel, S., \& Fröhlich, E. (2019). Outcomes of three different ways to train medical students as ultrasound tutors. BMC Medical Education, 19(1).

Chambers, B. R., Mack, J., Sabus, C., Becker, D., Shaw, P., \& Diederich, E. (2020). Interprofessional Procedure Training for Medicine and Nursing Students. MedEdPORTAL : The Journal of Teaching and Learning Resources, 16, 10884.

Crisp, D. A., Rickwood, D., Martin, B., \& Byrom, N. (2020). Implementing a peer support program for improving university student wellbeing: The experience of program facilitators. Australian Journal of Education, 64(2), 113-126.

Cross, R., Lowcock, D., Fiave, J., Agyeniwah, S., Annan, G. K., Cross, R., Lowcock, D., Fiave, J., Agyeniwah, S., \& Annan, G. K. (2020). "Feeling part of a network of learning in health promotion": An evaluation of a postgraduate peer-mentoring scheme in Ghana. INNOVATIONS IN EDUCATION AND TEACHING INTERNATIONAL, 57(2), 175-185.

Engels, D., Kraus, E., Obirei, B., \& Dethleffsen, K. (2018). Peer teaching beyond the formal medical curriculum. Advances in Physiology Education, 42(3), 439-448.

Evans, D. J. R., \& Cuffe, T. (2009). Near-peer teaching in anatomy: An approach for deeper learning. Anatomical Sciences Education, 2(5), 227-233. https://doi.org/10.1002/ase.110

Farkas, A. H., Allenbaugh, J., Bonifacino, E., Turner, R., \& Corbelli, J. A. (2019). Mentorship of US Medical Students: a Systematic Review. Journal of General Internal Medicine, 34(11), 2602-2609. https://doi.org/10.1007/s11606-019-05256-4

Franzoi, M. A. H., \& Martins, G. (2020). A mentoring experience among undergraduate nursing students: Dialogic reflections and resonances. Interface: Communication, Health, Education, 24, 1-15.

González L, R., Molina Z, H., García-Huidobro D, M., Stevens M, P., Jadue T, A., Riquelme U, A., Torres M, J., Alarcón O, F., Barra M, S., Toloza A, C., Ávalos T, M., \& Fasce H, E. (2019). Tutoría por pares de distinto año académico en la enseñanza de habilidades quirúrgicas básicas en estudiantes de pregrado de medicina TT - Peer tutoring in the teaching of basic surgical skills in medical undergraduate students. Rev. Cir. (Impr.), 71(6), 545-551.

Green, J. L. (2018). Peer support systems and professional identity of student nurses undertaking a UK learning disability nursing programme. Nurse Education in Practice, 30, 56-61.

Hossain, I., Mugoya, I., Muchai, L., Krudwig, K., Davis, N., Shimp, L., \& Richart, V. (2021). Blended learning using peer mentoring and WhatsApp for building capacity of health workers for strengthening immunization services in Kenya. Global Health Science and Practice, 9(1), 201-215. 
Krishna, L., Tay, K. T., Yap, H. W., Koh, Z. Y. K., Ng, Y. X., Ong, Y. T., Shivananda, S., Compton, S., Mason, S., Kanesvaran, R., Toh, Y. P., Krishna, L., Tay, K. T., Yap, H. W., Koh, Z. Y. K., Ng, Y. X., Ong, Y. T., Shivananda, S., Compton, S., ... Toh, Y. P. (2020). Combined novice, near-peer, e-mentoring palliative medicine program: A mixed method study in Singapore. PLOS ONE, 15(6).

Lapp, H., Makowka, P., Recker, F., Lapp, H., Makowka, P., \& Recker, F. (2018). Peer-mentoring Program during the Preclinical Years of Medical School at Bonn University: a Project Description. GMS JOURNAL FOR MEDICAL EDUCATION, 35(1).

Lemos, R. S., Bentes, L. G. de B., \& Brito, C. V. B. (2020). O método mentoring como suporte para a saúde mental na graduação em medicina. Pará Research Medical Journal, 4. https://doi.org/10.4322/prmj.2020.006

Lewis, V., Martina, C. A., McDermott, M. P., Chaudron, L., Trief, P. M., LaGuardia, J. G., Sharp, D., Goodman, S. R., Morse, G. D., \& Ryan, R. M. (2017). Mentoring Interventions for Underrepresented Scholars in Biomedical and Behavioral Sciences: Effects on Quality of Mentoring Interactions and Discussions. CBE-Life Sciences Education, 16(3), ar44. https://doi.org/10.1187/cbe.16-07-0215

Lewis, V., Martina, C. A., McDermott, M. P., Trief, P. M., Goodman, S. R., Morse, G. D., LaGuardia, J. G., Sharp, D., \& Ryan, R. M. (2017). A Randomized Controlled Trial of Mentoring Interventions for Underrepresented Minorities. Academic Medicine, 91(7), 994-1001. https://doi.org/10.1097/ACM.0000000000001056

Lockspeiser, T. M., O’Sullivan, P., Teherani, A., \& Muller, J. (2008). Understanding the experience of being taught by peers: the value of social and cognitive congruence. Advances in Health Sciences Education, 13(3), 361-372. https://doi.org/10.1007/s10459-006-9049-8

Loda, T., Erschens, R., Nikendei, C., Giel, K., Junne, F., Zipfel, S., \& Herrmann-Werner, A. (2020). A novel instrument of cognitive and social congruence within peer-assisted learning in medical training: Construction of a questionnaire by factor analyses. BMC Medical Education, 20(1).

Loda, T., Erschens, R., Nikendei, C., Zipfel, S., \& Herrmann-Werner, A. (2020). Qualitative analysis of cognitive and social congruence in peer-assisted learning-The perspectives of medical students, student tutors and lecturers. Medical Education Online, 25(1).

Masterson Creber, R. M., Baldwin, M. R., Brown, P. J., Rao, M. K., Goyal, P., Hummel, S., Dodson, J. A., Helmke, S., \& Maurer, M. S. (2019). Facilitated Peer Mentorship to Support Aging Research: A RE-AIM Evaluation of the CoMPAdRE Program. Journal of the American Geriatrics Society, 67(4), 804-810.

Menezes, D. P. F., Cunha, A. T. R. da, Oliveira, L. C. da R., \& Souza, L. F. de F. (2021). Peer mentoring como estratégia de acolhimento ao estudante e adaptação ao método PBL. Revista Brasileira de Educação Médica, 45(suppl 1). https://doi.org/10.1590/1981-5271v45.supl.1-20210088

Mota, A. M., Gomides, M. M., Costa, J. L., Pires, A. T., \& Carvalho, I. G. M. de. (2019). Mentoring E Educação Em Saúde. Facit Business and Technology Journal, 9(1), 147-153.

Naeger, D. M., Conrad, M., Nguyen, J., Kohi, M. P., \& Webb, E. M. (2013). Students Teaching Students. Academic Radiology, $20(9)$, 1177-1182. https://doi.org/10.1016/j.acra.2013.04.004

Ntho, T. A., Pienaar, A. J., \& Sehularo, L. A. (2020). Peer-mentees' challenges in an undergraduate peer-group clinical mentoring programme in a nursing education institution. Health SA Gesondheid, 25, 1-8.

Olaussen, A., Reddy, P., Irvine, S., \& Williams, B. (2016). Peer-assisted learning: time for nomenclature clarification. Medical Education Online, 21(1), 30974. https://doi.org/10.3402/meo.v21.30974

Page, M. J., McKenzie, J. E., Bossuyt, P. M., Boutron, I., Hoffmann, T. C., Mulrow, C. D., Shamseer, L., Tetzlaff, J. M., Akl, E. A., Brennan, S. E., Chou, R., Glanville, J., Grimshaw, J. M., Hróbjartsson, A., Lalu, M. M., Li, T., Loder, E. W., Mayo-Wilson, E., McDonald, S., ... Moher, D. (2021). The PRISMA 2020 statement: an updated guideline for reporting systematic reviews. BMJ, n71. https://doi.org/10.1136/bmj.n71

Patel, M., Aitken, D., Xue, Y. L., Sockalingam, S., Simpson, A., Patel, M., Aitken, D., Xue, Y., Sockalingam, S., \& Simpson, A. (2021). An evaluation of cascading mentorship as advocacy training in undergraduate medical education. BMC MEDICAL EDUCATION, 21(1).

Pethrick, H., Nowell, L., Oddone Paolucci, E., Lorenzetti, L., Jacobsen, M., Clancy, T., \& Lorenzetti, D. (2020). Peer mentoring in medical residency education: A systematic review. Canadian Medical Education Journal. https://doi.org/10.36834/cmej.68751

Phipps, W., Kansiime, R., Stevenson, P., Orem, J., Casper, C., \& Morrow, R. A. (2018). Peer Mentoring at the Uganda Cancer Institute: A Novel Model for Career Development of Clinician-Scientists in Resource-Limited Settings. Journal of Global Oncology, 4(4), 1-11. https://doi.org/10.1200/JGO.17.00134

Priest, H. M. (2006). Essentials of nursing research: methods, appraisal, and utilization Denise F Polit Essentials of nursing research: methods, appraisal, and utilization, Cheryl Tatano Beck Lipincott Williams and Wilkins $554 £ 24.950781749727$ 0781749727. Nurse Researcher, 13(4), 91-92. https://doi.org/10.7748/nr.13.4.91.s11

Prunuske, A., Houss, B., Kosobuski, A. W., Prunuske, A., Houss, B., \& Kosobuski, A. W. (2019). Alignment of roles of near-peer mentors for medical students underrepresented in medicine with medical education competencies: a qualitative study. BMC MEDICAL EDUCATION, 19(1).

Rompolski, K., \& Dallaire, M. (2020). The Benefits of Near-Peer Teaching Assistants in the Anatomy and Physiology Lab: An Instructor and a Student's Perspective on a Novel Experience. Journal of the Human Anatomy and Physiology Society, 24(1), 82-94.

Sambunjak, D., Straus, S. E., \& Marušić, A. (2006). Mentoring in Academic Medicine. JAMA, 296(9), 1103. https://doi.org/10.1001/jama.296.9.1103

Scott, S. E., Cook, S., Farmer, M. A., Kim, S. K., Pomfret, R. W., Samardzic, K., Hartzell, J. D., \& Hutchinson, J. W. (2019). The rising physicians program: A novel approach for mentoring medical students. Military Medicine, 184(5), e164-e167.

Smith, C. J., Matthias, T., Beam, E., Wampler, K., Pounds, L., Nickol, D., Carlson, K., \& Michael, K. (2018). Building a bigger tent in point-of-care ultrasound education: A mixed-methods evaluation of interprofessional, near-peer teaching of internal medicine residents by sonography students. BMC Medical Education, 18(1). 
Soares, C. B., Hoga, L. A. K., Peduzzi, M., Sangaleti, C., Yonekura, T., \& Silva, D. R. A. D. (2014). Integrative Review: Concepts And Methods Used In Nursing. Revista Da Escola de Enfermagem Da USP, 48(2), 335-345. https://doi.org/10.1590/S0080-6234201400002000020

Souza, M. G. de, Reato, L. de F. N., \& Bellodi, P. L. (2020). Ressignificando a Relação entre Calouros e Veteranos: Mentoria de Pares na Visão de Alunos Mentores TT - Giving New Meaning to the Relationship Between Freshmen and Seniors: Peer Mentoring in the View of Student Mentors. Rev. Bras. Educ. Méd, 44(4), e174-e174.

Tamachi, S., Giles, J. A., Dornan, T., \& Hill, E. J. R. (2018). “you understand that whole big situation they're in”: Interpretative phenomenological analysis of peer-assisted learning. BMC Medical Education, 18(1).

Ten Cate, O., \& Durning, S. (2007). Peer teaching in medical education: twelve reasons to move from theory to practice. Medical Teacher, 29(6), 591-599. https://doi.org/10.1080/01421590701606799

Terrion, J. L., \& Leonard, D. (2007). A taxonomy of the characteristics of student peer mentors in higher education: findings from a literature review. Mentoring \& Tutoring: Partnership in Learning, 15(2), 149-164. https://doi.org/10.1080/13611260601086311

Tsuei, S. H.-T., Lee, D., Ho, C., Regehr, G., \& Nimmon, L. (2019). Exploring the Construct of Psychological Safety in Medical Education. Academic Medicine: Journal of the Association of American Medical Colleges, 94(11), S28-S35.

Ursi, E. S., \& Gavão, C. M. (2006). Prevenção de lesões de pele no perioperatório: revisão integrativa da literatura. Revista Latino-Americana de Enfermagem, 14(1), 124-131. https://doi.org/10.1590/S0104-11692006000100017

Wareing, M., Green, H., Burden, B., Burns, S., Beckwith, M. A. R., Mhlanga, F., Mann, B., Wareing, M., Green, H., Burden, B., Burns, S., Beckwith, M. A. R., Mhlanga, F., \& Mann, B. (2018). "Coaching and Peer-Assisted Learning" (C-PAL) - The mental health nursing student experience: A qualitative evaluation. JOURNAL OF PSYCHIATRIC AND MENTAL HEALTH NURSING, 25(8), 486-495.

Weavind, L., \& Jobin McGrane, T. (2018). Women Mentoring Women in Academic Medicine: Pathways to Success. International Anesthesiology Clinics, 56(3), 110-120. https://doi.org/10.1097/AIA.0000000000000192

Zibold, J., Gernert, J. A., Reik, L. J. U., Keidel, L. M., Graupe, T., Dimitriadis, K., Zibold, J., Gernert, J. A., Reik, L. J. U., Keidel, L. M., Graupe, T., \& Dimitriadis, K. (2021). Adaptations to mentoring and peer mentor training at the medical faculty during the COVID-19 pandemic. GMS JOURNAL FOR MEDICAL EDUCATION, 38(1). 